\title{
Postprandial glycaemic responses in pre- and post-menopausal women
}

\author{
M. Sayegh, J.E. Drew and V. Ranawana \\ The Rowett Institute, University of Aberdeen, Foresterhill, Aberdeen, AB25 2ZD.
}

Postprandial hyperglycaemia increases diabetic and cardiovascular disease risk in healthy individuals ${ }^{(1)}$. Co-ingesting vegetables with high carbohydrate meals can attenuate postprandial glycaemic responses $(\mathrm{GR})^{(2)}$. This study aims to further investigate the effect of age and menstrual status on postprandial GR in pre- and post-menopausal women.

Eighteen, pre- $(\mathrm{n}=7)$ and post-menopausal $(\mathrm{n}=11)$ women $\left(\right.$ BMI $\left.25.2-30.4 \mathrm{~kg} / \mathrm{m}^{2}\right)$ have been recruited to The impact of Vegetables on acute glycaemia and Glycaemia induced CVD risk in women: metabolic effects and Inter-individual variations study (VegGI). Postprandial GR following rice (75 g of available carbohydrate) (R), rice with bok choy (150g) (RB) or spinach (150g) (RS) was measured every 5 minutes for 4 hours using a Continuous Glucose Monitoring system (Medtronic Ltd). Anthropometric, cholesterol (Cholestech LDX, Alere) and HbAlc (Afinion AS100, Alere) measures were made in a fasted state. Oestradiol, progesterone, follicular stimulating hormone (FSH) and testosterone were measured (ADVIA Centaur). Principal component analysis (PCA) (Umetrics SIMCA-P + 12.0) was performed on GR data. Total GR was expressed as the incremental area under the curve (iAUC) and calculated geometrically. A linear mixed model was used to analyse the impact of R, RB, RS on GR using age and diet as factors. Independent samples t-test was used for further data comparisons (ver.24.0; SPSS Inc).

The post-menopausal group was significantly older with significantly higher HbAlc, systolic BP, total cholesterol, FSH levels and lower oestradiol levels than pre-menopausal $(\mathrm{P}<0.05)$. PCA ( $\mathrm{PC} 1$ and $\mathrm{PC} 2)$ accounting for approximately $82 \%$ of the variation indicated age and hormonal status as factors contributing to postprandial GR rather than meal consumption. No significant difference was observed in postprandial GR with consumption of vegetables with the high carbohydrate meal (R) in either group. Inter-individual variation in postprandial GR was observed in both groups, with greatest variation observed in the post-menopausal group. Mean postprandial GR was significantly higher in the post-menopausal group following consumption of RB $(\mathrm{P}<0.045)$ (Fig 1A). A linear mixed model revealed a significant impact of meals on postprandial GR associated with age $(\mathrm{P}=0.010)$. The postmenopausal group had significantly higher postprandial GR following RB at $40-90$ minutes (Fig 1B).

(a)

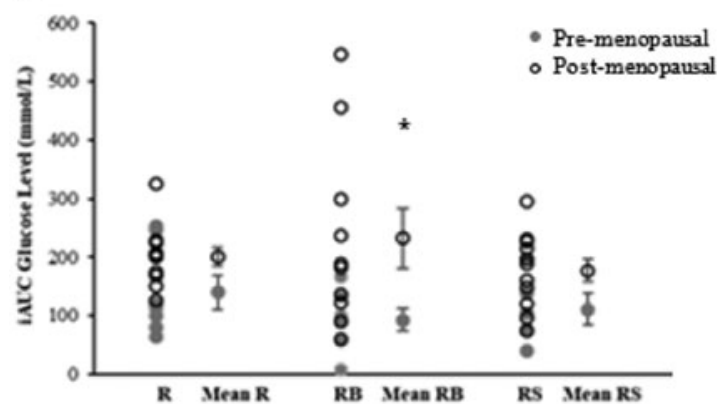

(b)

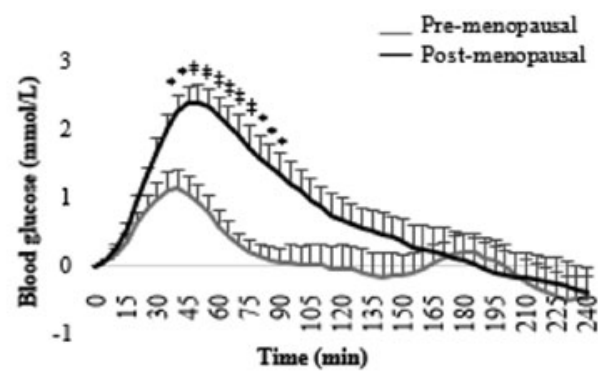

Fig. 1. $[A]$ iAUC distribution for postprandial GR in pre- $(R, R B n=7, R S n=5)$ and post-menopausal $(R n=11, R B n=10$, $R S n=11)$ individuals and mean values $\pm \mathrm{SE}$ of groups. [B] Mean change $\pm \mathrm{SE}$ in postprandial GR following consumption of $\mathrm{RB}$ using mixed linear model. ${ }^{*} \mathrm{P}<0.05 ;{ }^{*} \mathrm{P} \leq$ 0.001

Preliminary analysis of data suggests a difference in glucose metabolism regulation between pre- and post-menopausal women, but no significant reduction in postprandial GR was associated with co-ingesting vegetables with a high carbohydrate meal. There was an indication that postprandial GR varied more in the post-menopausal group. Recruitment is ongoing to increase group size and further assess factors influencing GR responses in pre- and post-menopausal women.

This work was supported by Scottish Government's Rural and Environment Science and Analytical Services Division

1. Woerle HJ, Neumann C, Zschau S et al. (2007) Diabetes Res Clin Pract 77, 280-285.

2. Kondo S, Suzuki A, Kurokawa M et al. (2016) Biomed Rep 5, 553-558. 